Article

\title{
External Control of Dissipative Coupling in a Heterogeneously Integrated Photonic Crystal-SOI Waveguide Optomechanical System
}

\author{
Viktor Tsvirkun ${ }^{1,+}{ }^{\text {, Alessandro Surrente }}{ }^{1}$, Fabrice Raineri ${ }^{1,2}{ }^{,}$Grégoire Beaudoin ${ }^{1}$, Rama Raj ${ }^{1}$, \\ Isabelle Sagnes ${ }^{1}$, Isabelle Robert-Philip ${ }^{1}$ and Rémy Braive ${ }^{1,2, *}$ \\ 1 Laboratoire de Photonique et Nanostructures LPN-CNRS UPR-20, Route de Nozay, \\ 91460 Marcoussis, France; victor.tsvirkun@gmail.com (V.T.); alessandro.surrente@gmail.com (A.S.); \\ fabrice.raineri@lpn.cnrs.fr (F.R.); gregoire.beaudoin@lpn.cnrs.fr (G.B.); rama.raj@lpn.cnrs.fr (R.R.); \\ isabelle.sagnes@lpn.cnrs.fr (I.S.); isabelle.robert@lpn.cnrs.fr (I.R.-P.) \\ 2 Université Paris Diderot, F-75205 Paris CEDEX 13, France \\ * Correspondence: remy.braive@lpn.cnrs.fr; Tel.: +33-1-6963-6049 \\ † Current address: Aix Marseille Univ., CNRS, Centrale Marseille, Institut Fresnel, 13013 Marseille, France.
}

Received: 31 August 2016; Accepted: 29 September 2016; Published: 12 October 2016

\begin{abstract}
Cavity optomechanical systems with an enhanced coupling between mechanical motion and electromagnetic radiation have permitted the investigation of many novel physical effects. The optomechanical coupling in the majority of these systems is of dispersive nature: the cavity resonance frequency is modulated by the vibrations of the mechanical oscillator. Dissipative optomechanical interaction, where the photon lifetime in the cavity is modulated by the mechanical motion, has recently attracted considerable interest and opens new avenues in optomechanical control and sensing. In this work we demonstrate an external optical control over the dissipative optomechanical coupling strength mediated by the modulation of the absorption of a quantum dot layer in a hybrid optomechanical system. Such control enhances the capability of tailoring the optomechanical coupling of our platform, which can be used in complement to the previously demonstrated control of the relative (dispersive to dissipative) coupling strength via the geometry of the integrated access waveguide.
\end{abstract}

Keywords: optomechanics; cavity optomechanics; photonic crystals; quantum dots

\section{Introduction}

Semiconductor nano-optomechanical systems have shown a rapid progress over the last years, with the demonstration of record optomechanical couplings, mechanical frequencies in the $\mathrm{GHz}$ range and above, and a well-controlled optical and mechanical dissipation [1]. For most of these systems, optomechanical coupling originates from a dispersive dependence of the nanocavity resonance frequency on its geometry, which is modulated by mechanical motion. Dissipative optomechanical coupling consists in the modulation of the lifetime of the cavity photons through the motion of a mechanical oscillator. Very recently, this effect has been observed in a wide variety of devices [2-7]. Dissipative coupling may significantly enhance the detection sensitivity in optomechanically-based sensing schemes [8,9]. Moreover, it could open new possibilities in the optomechanical control of systems featuring both types of coupling mechanisms [10,11], where a tailored coupling strength is highly desirable. Such tailoring was demonstrated using external [3] or integrated [6] variation of the geometry of the optical access channel to the nanocavity. These demonstrations, however, were based on the simultaneous modification of both types of couplings. Here we propose the possibility of exerting an external optical control exclusively of the intrinsic dissipative component of the optomechanical coupling within a hybrid optomechanical system by making use of a layer 
of semiconductor quantum dots (QDs). By introducing a non-resonant pump source, we achieve a deterministic modulation of the optical losses of the cavity induced by the QD absorption at frequencies close to the cavity resonance. We show that this optical modulation is sensitive to the mechanical motion of the device, coupled to the optical cavity resonance via an optomechanical interaction. Non-resonant external pumping of the QDs can saturate their absorption at wavelengths close to the cavity resonance, thus lowering or eliminating one of the intrinsic loss mechanisms of the cavity. The intrinsic dissipative coupling can therefore be controlled externally, deterministically and independently of the other coupling mechanisms. This can lead to a straightforward implementation of optomechanical resonators capable of reaching the optimal mixed coupling ratio in view of achieving an optimal optical cooling of the mechanical mode [11].

\section{Materials and Methods}

\subsection{Device Fabrication}

Silicon-on-insulator (SOI) access waveguide network, employed to couple light into and out of the photonic crystal (PhC) cavities, consists of $220 \mathrm{~nm}$ thick Si ridges, separated from the host Si substrate by a $2 \mu \mathrm{m}$ thick buried $\mathrm{SiO}_{2}$ layer to ensure good confinement of the guided mode. Each sample features a set of waveguides of variable width $(250 \mathrm{~nm}$ to $550 \mathrm{~nm})$ ensuring single mode TE-polarised operation around $1560 \mathrm{~nm}$ wavelength. Single waveguide length is $6 \mathrm{~mm}$. The light is coupled into and out from the waveguide using grating couplers [12] at its extremities. A $260 \mathrm{~nm}$ thick InP membrane layer, incorporating a single layer of self-assembled InAs(P) QDs in the middle, is grown on an $\operatorname{InP}(100)$ substrate using MetalOrganic Vapour Phase Epitaxy. The sample is capped with a $1 \mu \mathrm{m}$ thick InGaAs layer, employed as an etch-stop layer. The Si and III-V substrates are adhesively bonded by employing a $200 \mathrm{~nm}$ thick $\mathrm{SiO}_{2}$ buffer layer, deposited onto the $\mathrm{InP}$ membrane, and a $250 \mathrm{~nm}$ thick DiVinylSiloxane-BenzoCycloButene (DVS-BCB) layer, deposited onto the SOI substrate. A residual $30 \mathrm{~nm}$ thick layer of cured DVS-BCB on top of the planarized Si waveguide layer is left after the completion of the bonding process (the two substrates are pressed together for $60 \mathrm{~min}$ at $320^{\circ} \mathrm{C}$ ). Next, the InP substrate and the InGaAs etch-stop layer are removed via wet etching, exposing the $\mathrm{InP}$ membrane. Pattern transfer of the PhC and mesa structures onto the latter is achieved via electron-beam lithography (EBL), reactive ion etching and inductively coupled plasma etching. The SOI waveguides and the PhC cavities are aligned making use of alignment markers present in the waveguide level, which ensured an alignment accuracy of $\sim 40 \mathrm{~nm}$ [13]. The excess InP membrane is removed by dry etching, with a layer of EBL resist which protects the PhC cavities and the supporting structures. The PhC membranes are suspended after wet chemical etching of the underlying $\mathrm{SiO}_{2}$ and subsequent critical point drying.

\subsection{Photonic Crystal Design}

Defect $\mathrm{L}_{3}$ PhC cavities are obtained by omitting three holes in a line of an otherwise perfect hexagonal lattice (lattice constant $a=420 \mathrm{~nm}$ ). The geometrical parameters of the $\mathrm{PhC}$ lattice and the cavities were designed using a commercial software from Lumerical Solutions, Inc., Vancouver, BC, Canada, implementing the 3D Finite Difference in Time Domain (FDTD) method. The $\mathrm{L}_{3}$ cavity mode wavelength was adjusted to approximately $1560 \mathrm{~nm}$, resulting in PhC hole radii of $r \approx 100 \mathrm{~nm}$ [14]. A hole at each end of the cavity was shifted outwards by $d=0.2 a$ to enhance the optical quality factor $Q$ [15]. A $Q \sim 10^{4}$ of the unloaded cavities (in the absence of the access waveguides) has been measured by micro-photoluminescence $(\mu \mathrm{PL})$ spectroscopy [16]. 


\section{Results}

\subsection{System Description}

The device consisting of a 2D PhC lattice etched into a thin InP membrane and embedding a defect $\mathrm{L}_{3} \mathrm{PhC}$ cavity is shown in Figure 1a. The membrane is suspended with four bridges, connected to suspension pads at two sides, over a SOI waveguide in order to achieve a deterministic evanescent coupling of the guided light to and from the embedded cavity (Figure 1a). For full details on $\mathrm{PhC}$ structure design and the device fabrication procedure, see Materials and Methods. A layer of Stranski Krastanov QDs is incorporated in the centre of the membrane as the embedded light emitters (Figure 1b,c). Such InAs(P) QDs are suited for efficient non-resonant pumping below the InP gap with subsequent broadband emission in the near infrared range (Figure 1d). The PL spectrum of these QDs, filtered by the PhC cavity, provides information on the characteristics of the fabricated devices. For this purpose, we perform $\mu$ PL measurements by non-resonantly pumping the QDs with a continuous wave $(\mathrm{CW})$ laser $\left(\lambda_{\text {pump }}=800 \mathrm{~nm}\right)$. Emission from the PhC cavity is collected with a spectrograph (resolution 0.8-0.9 nm). Typical $\mu$ PL spectra, demonstrating the QD emission from an unpatterned area of the sample and from an $\mathrm{L}_{3}$ cavity, are displayed in Figure 1d. Pronounced peaks corresponding to band edge and cavity modes are well visible. The position of the excitation spot (typical size $3 \mu \mathrm{m}$ ) is indicated in Figure 1a. We notice a decrease of the loaded optical $Q$ of the cavity by about about a factor of 10 due to the evanescent coupling to the SOI waveguide.

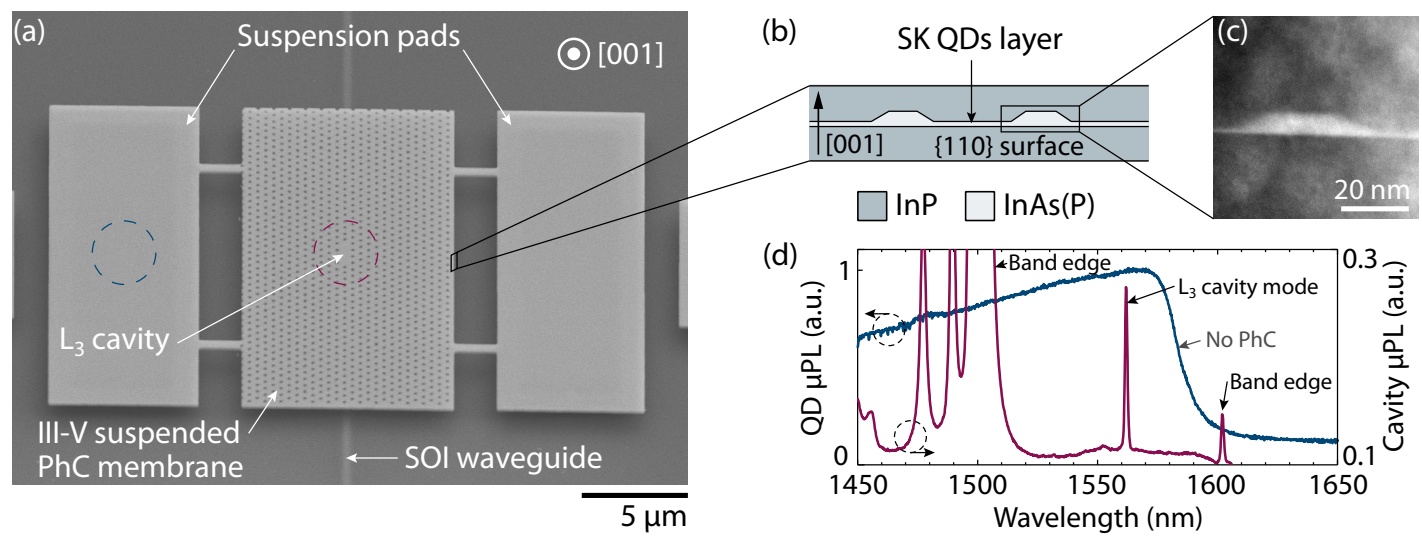

Figure 1. (a) SEM image of the fabricated device; (b) Schematic of the membrane cross-section composition (not to scale); (c) TEM image of a single QD; (d) $\mu \mathrm{PL}$ spectra of the QD layer from unpatterned membrane (dark blue) and from the region embedding an $\mathrm{L}_{3} \mathrm{PhC}$ cavity (violet).

\subsection{QDs Response to a Non-Resonant Optical Pump}

InAs(P) QDs show optical absorption around $1550 \mathrm{~nm}(0.8 \mathrm{eV})$ by two-photon absorption. This increases the optical cavity losses and broadens its linewidth, which is resonantly probed in our optomechanical experiments. By saturating the active medium using the non-resonant pump laser it is possible to control such absorption (and the associated cavity linewidth), as we show in the following.

Initially, we study the emission properties of the QDs in a $\mathrm{PhC}$ cavity coupled to an access waveguide. Figure 2a describes the optical set-up, where for this purpose we only use a CW pump laser $\left(\lambda_{\text {pump }}=800 \mathrm{~nm}\right)$, focused on the cavity surface by a microscope objective. The QD luminescence, filtered by the cavity and partially coupled to the SOI waveguide, is read out by the spectrometer after being outcoupled from one of the waveguide grating couplers through a microscope objective.

Although lasing from similar structures has been demonstrated at room temperature [17], we did not observe any kink in the light-in-light-out (L-L) curve under CW excitation (top graph in Figure 2b), suggesting that the lasing threshold is not reached in the current configuration, and the measured 
signal consists exclusively of the spontaneous emission of the active medium. This can be explained by additional losses of the optical cavity mode, induced by the presence of the access waveguide in its evanescent field. The linewidth of the cavity resonance decreases significantly (see bottom graph in Figure $2 b$ ) as the pump power is increased, demonstrating the progressive saturation of the QD absorption and consequently the decrease of the optical losses due to two-photon absorption. At high pump power (middle graph in Figure $2 b$ ) the emission wavelength red shifts, owing to thermal effects. Finally, a thermal degradation of the QD emission is observed at pump power larger than $1 \mathrm{~mW}$ with a simultaneous increase of the emission linewidth (bottom graph in Figure $2 \mathrm{~b}$ ) due to thermal fluctuations.

(a)

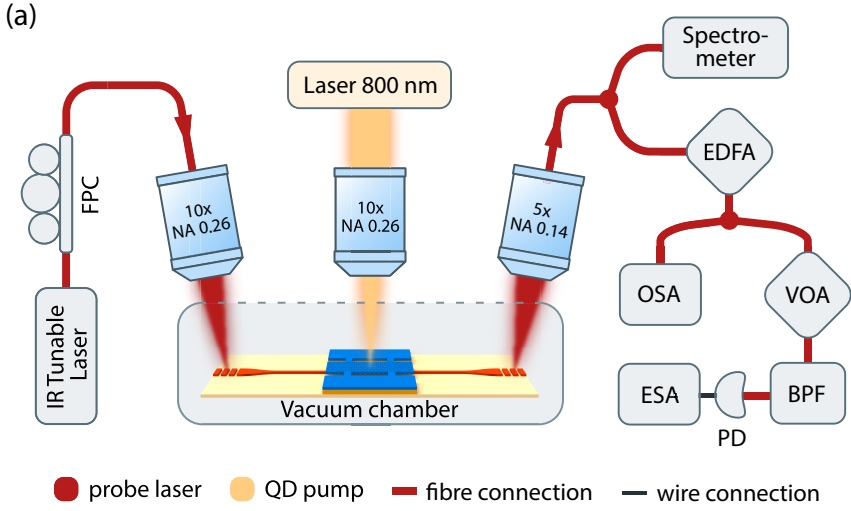

(b)

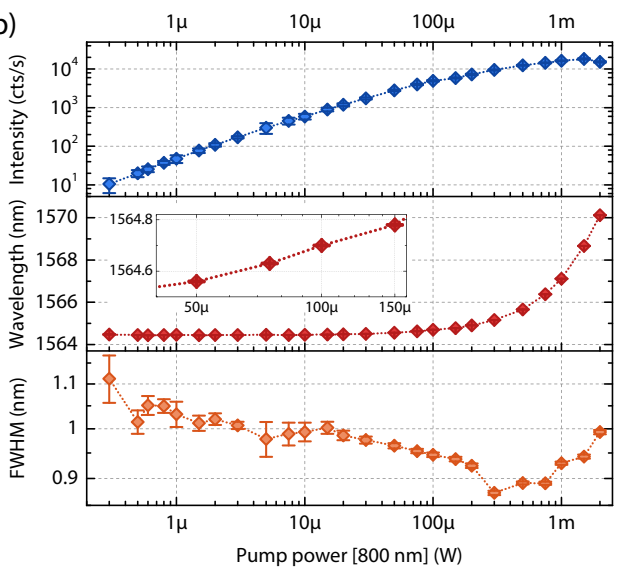

Figure 2. (a) Schematic of the experimental set-up for the characterization of optical/optomechanical transduction of non-resonant pump $\left(\lambda_{\text {pump }}=800 \mathrm{~nm}\right)$ to resonant probe $\left(\lambda_{\text {probe }} \approx 1.56 \mu \mathrm{m}\right)$; (b) Emission properties of the QDs, filtered by the $\mathrm{L}_{3}$ cavity (loaded $\lambda_{\mathrm{c}}=1564.5 \mathrm{~nm}$ ): fitted intensity $I$, wavelength $\lambda_{0}$ and linewidth $\kappa$ of the collected emission are plotted versus non-resonant pump power. Inset: zoom-in of $\lambda_{0}$ power dependence.

These measurements indicate that within a certain range of laser pump powers $P_{\text {pump }}$ the derivatives $g_{\omega, \mathrm{QD}}=\mathrm{d} \omega_{0} / \mathrm{d} P_{\text {pump }}$ and $g_{\kappa, \mathrm{QD}}=\mathrm{d} \kappa_{t} / \mathrm{d} P_{\text {pump }}$ are non-zero (e.g., see the inset in Figure $2 b$ ), therefore we can establish an analogy with dispersive and dissipative coupling coefficients for the mechanical mode [3], where the modulation of the membrane coordinate is replaced by the modulation of $P_{\text {pump }}$ power. For an excitation power of $P_{\text {pump }}=100 \mu \mathrm{W}$ (excitation level at which no thermal effects degrading the QD emission are observed), the modulation coefficients are estimated to be $g_{\omega, \mathrm{QD}} \approx 275 \mathrm{MHz} / \mu \mathrm{W}$ and $g_{\kappa, \mathrm{QD}} \approx 33.5 \mathrm{MHz} / \mu \mathrm{W}$.

\subsection{Optomechanical Transduction of Non-Resonant Pump}

In our recent work [6], we reported on tailored dispersive and dissipative optomechanical couplings for the flexural mechanical modes of our devices. Here we investigate the impact of the embedded active medium on the optomechanical coupling by using an external modulation of the pump laser. To detect these effects, we employ the side-of-the-fringe technique, commonly used in optomechanical experiments for the detection of Brownian motion [1].

The experimental set-up is sketched in Figure 2a. We couple a probe laser signal into the SOI waveguide, the laser beam is coupled to the heterogeneously integrated PhC cavity, transmitted through the access SOI waveguide and collected from the output grating coupler through a microscope objective. We employ a sharp probe $(\approx 200 \mathrm{kHz}$ linewidth) at fixed wavelength instead of the full spectrum of the laser. The outcoupled light first is selectively amplified by a two-stage erbium doped fibre amplifier (EDFA). The first stage of the EDFA is followed by a $20 \mathrm{GHz}$ wide spectral filter, locked to the probe laser resonance. The locking is controlled in real-time mode on an optical signal analyser (OSA; Anritsu MS9740A, Kanagawa, Japan), where a part of the amplified signal is directed (Figure 2a). 
This functionality allows us to perform wavelength sweep experiments while still ensuring stable signal amplification with high signal-to-noise ratios (SNR) and a noise figure at the level of state of the art EDFAs. Following the second path (Figure 2a), after amplification the probe signal power is adjusted by a variable optical attenuator (VOA; Thorlabs VOA50, Newton, NJ, USA) in order to match the working range of the detector input power. This probe signal is additionally filtered by the tunable (spectrally with variable linewidth) bandpass filter (BPF; Yenista WSM-160, Lannion, France), which allows to reduce the noise related to the modulation of the laser carrier at wavelengths different from that of the sharp probe (e.g., background of amplified spontaneous emission). The output signal of the fast photodiode (New Focus 1592, Irvine, CA, USA) is read out by the electric spectrum analyser (ESA; Rohde\&Schwarz FSV7, Munich, Germany).

The PhC cavity is additionally externally pumped with $\lambda_{\text {pump }}=800 \mathrm{~nm}$ CW laser at a pump power $P_{\text {pump }}=34 \mu \mathrm{W}$. A small $P_{\text {pump }}$ modulation with a sine function at $\Omega_{\text {mod }} / 2 \pi=5.65 \mathrm{MHz}$ is performed via the current modulation input of the pump laser diode controller (ILX Lightwave LDC-3724C, Bozeman, MT, USA) using a function generator. This modulation frequency is chosen close to the mechanical resonance frequency in order to have a comparable noise floor, but not too close (at least 10 mechanical linewidths away) to $\Omega_{\mathrm{m}}$ in order not to alter the optomechanical response. Taking into account the modulation depth and the corresponding transduction parameters (amplitude of $20 \mathrm{~V}_{\mathrm{pp}}$ from function generator; controller transduction $20 \mathrm{~mA} / \mathrm{V}$; calibrated laser diode responsivity $4.67 \mathrm{~mW} /$ A within the range of used $P_{\text {pump }}$ powers), the power output of the $800 \mathrm{~nm}$ pump laser is $P_{\text {pump }}=(34.00 \pm 0.93) \mu \mathrm{W}$.
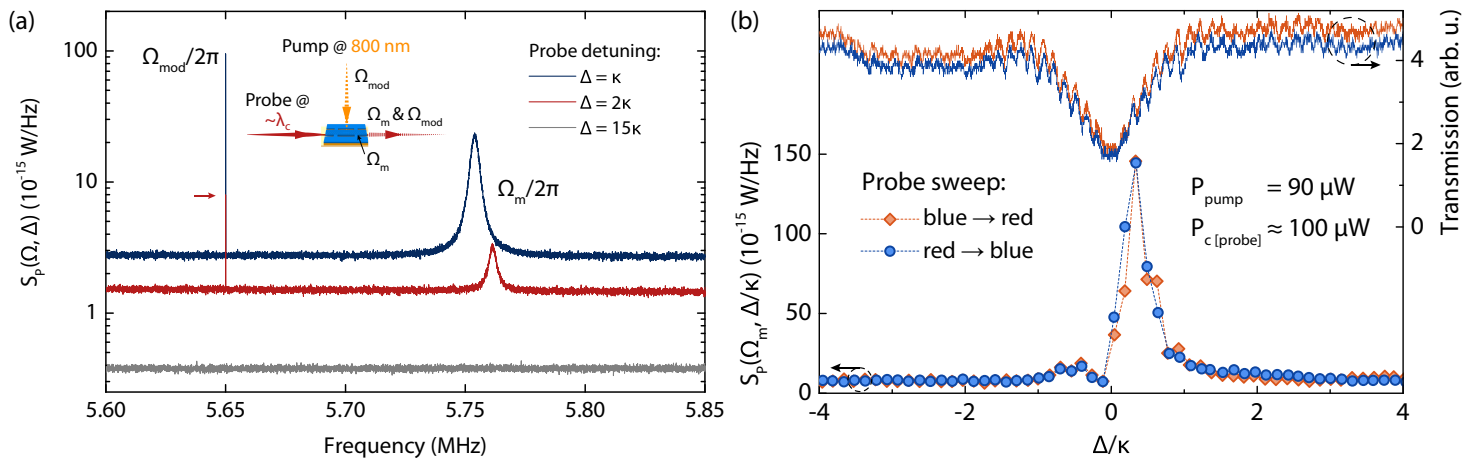

Figure 3. (a) Spectra of the probe light, as measured by the Electric spectrum analyser (ESA), coupled to the PhC cavity with a layer of QDs in the middle, pumped non-resonantly at normal incidence. The pump laser power is modulated at $\Omega_{\text {mod }} / 2 \pi$. Shown curves are measured at different laser-cavity detunings $\Delta=\lambda_{\text {probe }}-\lambda_{0}$. The Lorentzian peak at $\Omega_{\mathrm{m}} / 2 \pi$ corresponds to the fundamental flexural mode of the membrane (membrane size: $8.5 \times 4.8 \times 0.26 \mu \mathrm{m}$ ). Inset: schematic describing the origin of the two detected peaks; (b) (bottom) Mechanical $\mathrm{M}_{1}$ mode amplitude dependence on probe laser detuning, acquired for forward (blue- to red-detuned) and backward (red- to blue-detuned) scanning directions. (top) Waveguide transmission spectra for both probe sweep directions. Measurements in (b) were performed at the same probe laser power (corresponding to the intracavity power $P_{\mathrm{c}} \approx 100 \mu \mathrm{W}$ ) and pump laser power $P_{\text {pump }}=90 \mu \mathrm{W}$; at room temperature $T_{\mathrm{b}}=294 \mathrm{~K}$ and at low pressure $p<10^{-4}$ mbar.

The noise spectra of the transmitted probe light, coupled to the defect PhC cavity resonance, at different laser-cavity detunings $\Delta$ and close to the fundamental mode frequency of the membrane Brownian motion consists of two resonance peaks (Figure 3a): one corresponding to the fundamental mechanical mode $\left(\mathrm{M}_{1}\right)$ at $\Omega_{\mathrm{m}} / 2 \pi$ and another related to the modulation frequency $\Omega_{\bmod } / 2 \pi$ of the non-resonant pump laser. For the mechanical mode at $\Omega_{\mathrm{m}} / 2 \pi$ we observe the optical spring effect: the mechanical mode amplitude $S_{\mathrm{P}}\left(\Omega_{\mathrm{m}}\right)$, linewidth and frequency $\Omega_{\mathrm{m}}$ are modified when the laser probe is swept across the cavity optical resonance [6]. Concerning the modulation peak, it does not come from direct modulation of the probe laser carrier, but is transferred to it via the QD layer. 
The modulation of the QD absorption creates photons at $\Omega_{\text {mod }}$ which are filtered by the cavity and coupled to the waveguide at the probed wavelength. The incident power of the non-resonant pump in our experiments did not exceed $250 \mu \mathrm{W}$ (Figure $2 \mathrm{~b}$ ), to avoid possible concomitant thermal effects whose relaxation time is of the order of $1 \mu \mathrm{s}$ [18].

\subsection{Tailored Optomechanical Couplings}

We compare the amplitude dependence of the pump modulation peak, read out by the probe laser, with the evolution of the mechanical amplitude of the fundamental membrane mode by performing optical spring effect measurements. To confirm that the cavity resonance is not influenced by the resonant probe, we compare the mechanical response of $\mathrm{M}_{1}$ for two scanning directions of the probe laser (Figure 3b) for $P_{\text {probe }}$ power, used for the present experiments (corresponding to the intracavity power $P_{\mathrm{c}} \approx 100 \mu \mathrm{W}$ ). The two responses are almost identical with no abrupt jumps (small discrepancies are related to a slight drift of the sample position with respect to the incident probe beam during the measurement series: the acquisition time of a single curve shown in Figure $3 \mathrm{~b}$ (bottom) is a few hours), which confirms that no optical bi-stability is induced at these measurement conditions. This is confirmed by measuring the cavity response to the probe laser for both sweeping directions, as shown in Figure $3 b$ (top). In the following we will analyse the experimental data for one of the sweep directions only.
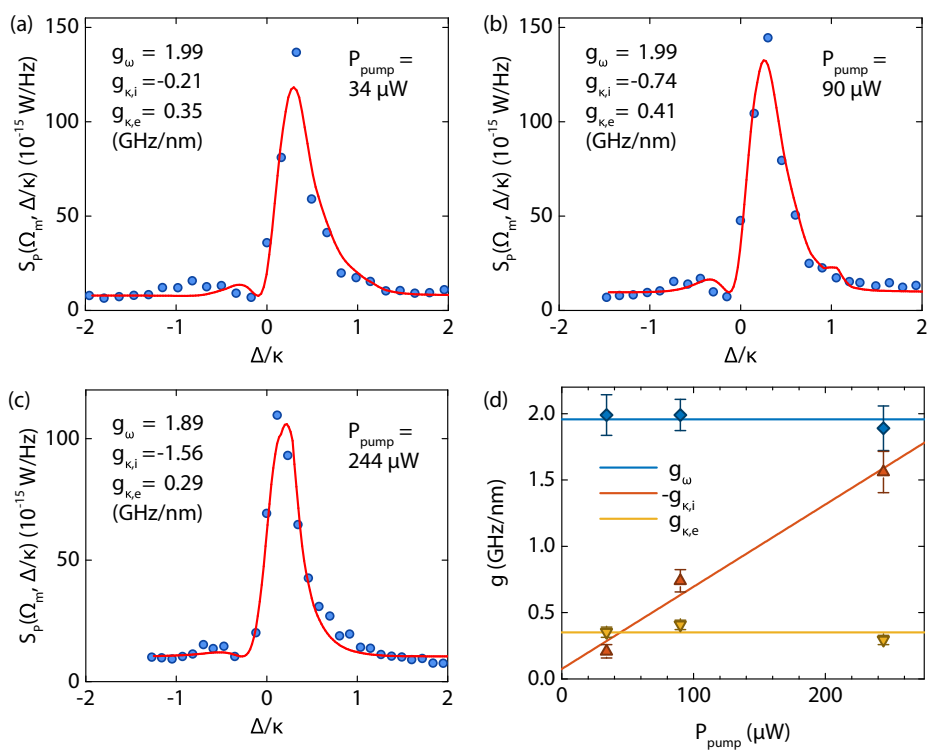

Figure 4. $(\mathbf{a}-\mathbf{c})$ Power spectral densities at mechanical resonance (circles: experimental data; solid lines: fit to model [6]) $S_{\mathrm{P}}\left(\Omega_{\mathrm{m}}, \Delta / \kappa\right)$ and fitted relative contributions of coupling dispersive $\left(g_{\omega}\right)$ and dissipative (intrinsic $g_{\kappa, \mathrm{i}}$ and external $g_{\kappa, \mathrm{e}}$ ) coupling strengths for different powers of non-resonant pump; (d) Experimental evolution of the optomechanical coupling coefficients with the power of the external non-resonant pump (markers-data from $(\mathbf{a}-\mathbf{c})$, solid lines-linear fits).

As discussed before, an increasing pump power results in the spectral narrowing of the cavity resonance (with its Lorentzian line shape preserved). We study the impact of this effect on the optomechanical coupling of the mechanical mode for several $P_{\text {pump }}$ values (the amplitude of the pump laser modulation is kept constant). Figure 4a-c display the experimental evolution of the fundamental mode amplitude as a function of the probe laser detuning for different powers of non-resonant $(800 \mathrm{~nm})$ pump laser. We have used an analytical model, describing this phenomenon by considering the impact of dissipative, intrinsic dispersive and external dispersive optomechanical couplings, with coupling coefficients $g_{\omega}, g_{\kappa, \mathrm{i}}$ and $g_{\kappa, \mathrm{e}}$, respectively [3,6]. The fitting procedure follows the one described in [6]: we took as initial guess the experimental coupling coefficients, measured for the fundamental mode 
and same access waveguide geometry in the absence of an external pump [6]. Subsequently, we let all the three coefficients free. The membrane resonator used in this experiment is smaller than the one described in [6], yielding a smaller value of the effective mass of the fundamental mechanical mode, estimated to be $m_{\text {eff }}=23.9 \mathrm{pg}$ [14]. The obtained fitted curves are displayed in Figure 4 along with the deduced optomechanical coupling coefficients for three values of external pump power: 34, 90 and $244 \mu \mathrm{W}$. As can be seen, an increase of $P_{\text {pump }}$ leads to a clear raise of the intrinsic dissipative coupling coefficient $g_{\kappa, \mathrm{i}}$ (from -0.21 to $-1.56 \mathrm{GHz} / \mathrm{nm}$ ), whilst the other components remain almost unchanged (Figure 4d).

\section{Discussion and Conclusions}

We have demonstrated hybrid optomechanical resonators, consisting of a thin InP membrane embedding a single layer of $\operatorname{InAs}(\mathrm{P})$ QDs and an $\mathrm{L}_{3} \mathrm{PhC}$ cavity, coupled to an integrated SOI waveguide. We show that by non-resonantly pumping the QD layer one can saturate the cavity losses due to the QD absorption and thus change the linewidth of the embedded cavity (Figure 2b). By monitoring the optomechanical response of the fundamental mechanical mode as a function of the probe laser wavelength, we demonstrated a control over the intrinsic dissipative coupling coefficient, which was shown to increase by a factor of 8 . The dispersive and external dissipative coupling coefficients remained unmodified (Figure 4d). Mechanisms yielding the observed transduction might include thermal effects, with characteristic times of the order of microseconds ( $\sim \mathrm{MHz}$ modulation frequencies) [19], or absorption and re-emission from the QDs, with characteristic times in the nanosecond range ( $\sim \mathrm{GHz}$ modulation frequencies). Radiation pressure is not considered here, as this effect is too weak for the low reflectivity of our system at the pump wavelength in the absence of an external cavity.

The studied phenomenon can be regarded as a way to finely tailor the dissipative optomechanical coupling strength in addition to the previously demonstrated tailored couplings in such resonators via changing the geometry of the access waveguides [6]. The ability to increase the dissipative coupling via the control of the QD absorption is beneficial for the presented device and could also be exploited in other optomechanical systems.

Acknowledgments: This work was supported by the Agence Nationale de la Recherche: programme ANR-2011-B504-028-01 MiNOToRe, the French RENATECH network, the European Marie Curie Initial Training Network: cQOM-Cavity Quantum Optomechanics, and the European Research Area Network (ERANet) project OMC.

Author Contributions: R.B., I.R.-P. and V.T. conceived and designed the experiments; V.T. designed the optomechanical resonators; G.B. and I.S. carried out the epitaxial growth runs; V.T., F.R. and R.B. fabricated the devices; V.T. performed the experiments; A.S. and V.T. analysed the data; V.T., A.S. and R.B. wrote the paper with extensive feedback from all co-authors; R.R., I.R.-P. and R.B. supervised the project.

Conflicts of Interest: The authors declare no conflict of interest.

\section{Abbreviations}

The following abbreviations are used in this manuscript:

$\begin{array}{ll}\text { CW } & \text { continuous wave } \\ \text { EBL } & \text { electron beam lithography } \\ \text { FDTD } & \text { finite difference in time domain } \\ \text { FWHM } & \text { indium arsenide phosphide } \\ \mathrm{InAs}(\mathrm{P}) & \text { indium gallium arsenide } \\ \mathrm{InGaAs} & \text { indium phosphide } \\ \mathrm{InP} & \text { photonic crystal } \\ \mathrm{PhC} & \text { micro-photoluminescence } \\ \mu \mathrm{PL} & \text { silicon } \\ \mathrm{Si} & \text { silicon dioxide } \\ \mathrm{SiO} & \text { quantum dot } \\ \mathrm{QD} & \end{array}$




\section{References}

1. Aspelmeyer, M.; Kippenberg, T.J. Cavity Optomechanics; Springer: Berlin/Heidelberg, Germany, 2014.

2. Li, M.; Pernice, W.H.P.; Tang, H.X. Reactive cavity optical force on microdisk-coupled nanomechanical beam waveguides. Phys. Rev. Lett. 2009, 103, 223901.

3. Wu, M.; Hryciw, A.C.; Healey, C.; Lake, D.P.; Jayakumar, H.; Freeman, M.R.; Davis, J.P.; Barclay, P.E. Dissipative and dispersive optomechanics in a nanocavity torque sensor. Phys. Rev. X 2014, 4, 021052.

4. Sawadsky, A.; Kaufer, H.; Nia, R.M.; Tarabrin, S.P.; Khalili, F.Y.; Hammerer, K.; Schnabel, R. Observation of generalized optomechanical coupling and cooling on cavity resonance. Phys. Rev. Lett. 2015, 114, 043601.

5. Hryciw, A.C.; Wu, M.; Khanaliloo, B.; Barclay, P.E. Tuning of nanocavity optomechanical coupling using a near-field fiber probe. Optica 2015, 2, 491-496.

6. Tsvirkun, V.; Surrente, A.; Raineri, F.; Beaudoin, G.; Raj, R.; Sagnes, I.; Robert-Philip, I.; Braive, R. Integrated III-V photonic crystal-Si waveguide platform with tailored optomechanical coupling. Sci. Rep. $2015,5,16526$.

7. Zhang, H.; Zeng, C.; Chen, D.; Li, M.; Wang, Y.; Huang, Q.; Xiao, X.; Xia, J. Femtogram scale nanomechanical resonators embedded in a double-slot photonic crystal nanobeam cavity. Appl. Phys. Lett. 2016, 108, 051106.

8. Davis, J.P.; Vick, D.; Fortin, D.C.; Burgess, J.A.J.; Hiebert, W.K.; Freeman, M.R. Nanotorsional resonator torque magnetometry. Appl. Phys. Lett. 2010, 96, 072513.

9. Forstner, S.; Prams, S.; Knittel, J.; van Ooijen, E.D.; Swaim, J.D.; Harris, G.I.; Szorkovszky, A.; Bowen, W.P.; Rubinsztein-Dunlop, H. Cavity optomechanical magnetometer. Phys. Rev. Lett. 2012, 108, 120801.

10. Elste, F.; Girvin, S.M.; Clerk, A.A. Quantum noise interference and backaction cooling in cavity nanomechanics. Phys. Rev. Lett. 2009, 102, 207209.

11. Weiss, T.; Nunnenkamp, A. Quantum limit of laser cooling in dispersively and dissipatively coupled optomechanical systems. Phys. Rev. A 2013, 88, 023850.

12. Taillaert, D.; van Laere, F.; Ayre, M.; Bogaerts, W.; van Thourhout, D.; Bienstman, P.; Baets, R. Grating couplers for coupling between optical fibers and nanophotonic waveguides. Jpn. J. Appl. Phys. 2006, 45, 6071-6077.

13. Karle, T.J.; Halioua, Y.; Raineri, F.; Monnier, P.; Braive, R.; Le Gratiet, L.; Beaudoin, G.; Sagnes, I.; Roelkens, G.; van Laere, F.; et al. Heterogeneous integration and precise alignment of InP-based photonic crystal lasers to complementary metal-oxide semiconductor fabricated silicon-on-insulator wire waveguides. J. Appl. Phys. 2010, 107, 063103.

14. Tsvirkun, V. Optomechanics in Hybrid Fully-Integrated Two-Dimensional Photonic Crystal Resonators. Ph.D. Thesis, Université Paris Sud-Paris XI, Orsay, France, 2015.

15. Akahane, Y.; Asano, T.; Song, B.S.; Noda, S. High-Q photonic nanocavity in a two-dimensional photonic crystal. Nature 2003, 425, 944-947.

16. Gavartin, E.; Braive, R.; Sagnes, I.; Arcizet, O.; Beveratos, A.; Robert, I. Optomechanical coupling in a two dimensional photonic crystal cavity. Phys. Rev. Lett. 2011, 106, 203902.

17. Hostein, R.; Braive, R.; Le Gratiet, L.; Talneau, A.; Beaudoin, G.; Robert-Philip, I.; Sagnes, I.; Beveratos, A. Demonstration of coherent emission from high-beta photonic crystal nanolasers at room temperature. Opt. Lett. 2010, 35, 1154-1156.

18. Moreau, V.; Tessier, G.; Raineri, F.; Brunstein, M.; Yacomotti, A.; Raj, R.; Sagnes, I.; Levenson, A.; de Wilde, Y. Transient thermoreflectance imaging of active photonic crystals. Appl. Phys. Lett. 2010, 96, 091103.

19. Usami, K.; Naesby, A.; Bagci, T.; Nielsen, B.M.; Liu, J.; Stobbe, S.; Lodahl, P.; Polzik, E.S. Optical cavity cooling of mechanical modes in a semiconductor nanomembrane. Nat. Phys. 2012, 8, 168-172.

(C) 2016 by the authors; licensee MDPI, Basel, Switzerland. This article is an open access article distributed under the terms and conditions of the Creative Commons Attribution (CC-BY) license (http://creativecommons.org/licenses/by/4.0/). 